\title{
BAIRE SECTIONS FOR GROUP HOMOMORPHISMS
}

\author{
S. GRAF AND G. MÄGERL
}

\begin{abstract}
The following result is proved: Let $X$ and $Y$ be compact topological groups and $p$ be a continuous group homomorphism from $Y$ onto $X$. Then there exists a map $q$ from $X$ to $Y$ such that $p \circ q=\operatorname{id}_{X}$ and $q^{-1}(B)$ is a Baire set in $Y$ for every Baire subset $B$ of $X$.
\end{abstract}

1. Introduction. As pointed out by Rieffel [7], Baire measurable sections for group homomorphisms can be used to construct certain well-behaved extension groups. This motivated Kupka [4] to ask the following question: Given a locally compact group $Y$, a closed subgroup $H$ of $Y$ and the canonical map $p$ from $Y$ onto the space $Y / H$ of left cosets of $Y$, does there exist a Baire measurable map $\varphi: Y / H \rightarrow Y$ with $p \circ \varphi=\mathrm{id}_{Y / H}$ ? We will show that the answer is "yes" provided $Y$ is compact and $H$ is a normal subgroup.

2. Preliminaries. Let $X$ and $Y$ be compact Hausdorff spaces, $\mathscr{B}_{0}(X)$ and $\mathscr{B}_{0}(Y)$ their respective Baire o-fields. A map $f: X \rightarrow Y$ is called Baire measurable iff $f^{-1}(B) \in \mathscr{B}_{0}(X)$ for all $B \in \mathscr{B}_{0}(Y)$. A map $\Phi$ from $X$ to the nonempty subsets of $Y$ is said to be a correspondence from $X$ to $Y$ (correspondences are also called multifunctions or set-valued functions in the literature). By $G(\Phi)$ we denote the graph $\{(x, y) \in X \times Y \mid y \in \Phi(x)\}$ of $\Phi$. $\Phi$ is called upper semi-continuous (u.s.c.) iff, for every open subset $U$ of $Y$, the set $\{x \in X \mid \Phi(x) \subset U\}$ is open in $X$. A compact-valued correspondence $\Phi$ is u.s.c. if and only if $G(\Phi)$ is closed in $X \times Y$.

A map $f: X \rightarrow Y$ is called a selection for $\Phi$ iff $f(x) \in \Phi(x)$ for all $x \in X$. A compact Hausdorff space $X$ is said to have the Bockstein separation property (BSP) iff any two disjoint open subsets of $X$ can be separated by open $\mathscr{F}_{\sigma}$-sets (cf. Pelczyński [6, Definition 5.9]). A classical theorem of Bockstein [1] states that an arbitrary product of compact metrizable spaces has the BSP. The same is true for compact topological groups (cf. Pelczyński [6, Theorem 7.5 and Corollary 5.11]).

3. A selection lemma. The following lemma will be used in the proof of our main theorem but may also be of some interest in itself.

LEMMA. Let $X$ be a compact Hausdorff space with the BSP, $Z$ a compact metrizable space, and $\Phi$ an u.s.c. compact-valued correspondence from $X$ to $Z$. Then $\Phi$ has a Baire measurable selection.

Received by the editors October 1, 1981 and, in revised form, February 9, 1982. 1980 Mathematics Subject Classification. Primary 54C65, 54C60, 28B20; Secondary 22C05.

Key words and phrases. Baire measurable selections, compact groups, Bockstein separation property.

(C)1982 American Mathematical Society 0002-9939/82/0000-0211/\$02.00 
Proof. We first note that, due to the fact that $X$ has the BSP, the following holds.

For every subset $F$ of $X$ the set $\stackrel{\circ}{F}$ is a Baire set

(where $\bar{A}$ and $\dot{A}$ denote the closure and the interior of a set $A$ respectively). To show this let $F$ be a subset of $X$. BSP implies that there is an open Baire set $B$ such that $\bar{F} \subset B$ and $B \cap(X \backslash \bar{F})=\varnothing$. This implies $\bar{F} \subset B \subset \bar{F}$, hence $\bar{F}=B$ because $B$ is open.

We will now show that there is a compact-valued correspondence $\tilde{\Phi}$ from $X$ to $Z$ such that

(i) $\tilde{\Phi}(x)$ is a subset of $\Phi(x)$ for all $x$ in $X$,

(ii) $\{x \in X \mid \tilde{\Phi}(x) \cap A \neq \varnothing\}$ is a Baire subset of $X$ for all closed subsets $A$ of $Z$.

Suppose for the moment that there is such a $\tilde{\Phi}$. Then the selection theorem of Kuratowski and Ryll-Nardzewski [5] implies that $\tilde{\boldsymbol{\Phi}}$ has a Baire measurable selection. Since such a selection is also a selection for $\Phi$ our lemma will follow.

To construct $\tilde{\Phi}$ we define for each $x$ in $X$ a collection $\mathscr{F}_{x}$ of nonempty subsets of $Z$ by

$$
\mathscr{F}_{x}:=\left\{B \subset Z \mid B \text { open, } x \in \overline{\Phi_{-1}(B)}\right\}
$$

where $\Phi_{-1}(B):=\{x \in X \mid \Phi(x) \subset B\}$. We claim that $\mathscr{F}_{x}$ has the finite intersection property. This is a consequence of the following facts:

(1) $\Phi_{-1}(F \cap G)=\Phi_{-1}(F) \cap \Phi_{-1}(G)$ for any two subsets $F$ and $G$ of $Z$,

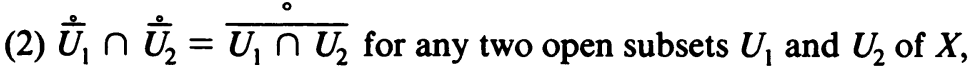

(3) $\Phi_{-1}(B)$ is open for every open subset $B$ of $Z$ because $\Phi$ is u.s.c.

Therefore $\tilde{\Phi}(x):=\cap\left\{\bar{B} \mid B \in \mathscr{F}_{x}\right\}$ defines a compact-valued correspondence $\tilde{\Phi}$ from $X$ to $Z$.

To show that $\tilde{\Phi}$ satisfies (i), assume that there are $x$ in $X$ and $z$ in $Z$ such that $z \in \tilde{\Phi}(x) \backslash \Phi(x)$. Because $Z$ is regular there is an open neighborhood $U$ of $z$ with $\bar{U} \cap \Phi(x)=\varnothing$. This implies $x \in \Phi_{-1}(Z \backslash \bar{U})$, hence $Z \backslash \bar{U} \in \mathscr{F}_{x}$ and therefore $z \in U$ $\cap \overline{(Z \backslash \bar{U})} \subset U \cap \overline{(Z \backslash U)}=U \cap(Z \backslash U)=\varnothing$ which is absurd.

(ii) is equivalent to

(ii) $\tilde{\Phi}_{-1}(U)$ is a Baire set for every open subset $U$ of $Z$.

So let $U \subset Z$ be open. Since $Z$ is metrizable there exists an increasing sequence $\left(B_{n}\right)_{n \in \mathrm{N}}$ of open sets such that $\cup_{n} B_{n}=\cup_{n} \bar{B}_{n}=U$. We show that

$$
\tilde{\Phi}_{-1}(U)=\bigcup_{n} \frac{\circ}{\Phi_{-1}\left(B_{n}\right)}
$$

holds, from which (ii)' will follow because each of the sets $\overline{\Phi_{-1}\left(B_{n}\right)}$ is a Baire set by $(*)$.

For $x \in \overline{\Phi_{-1}\left(B_{n}\right)}$ we have $B_{n} \in \mathscr{F}_{x}$, hence $\tilde{\Phi}(x) \subset \bar{B}_{n} \subset U$, which proves one of the required inclusions. To prove the other one let $\tilde{\Phi}(x)$ be contained in $U$. This 
implies that $\bar{B} \subset U$ holds for some $B \in \mathscr{F}_{x} . \bar{B}$ being compact there is an $n \in \mathbf{N}$ with $\bar{B} \subset B_{n}$. Therefore $x \in \overline{\Phi_{-1}(B)} \subset \overline{\Phi_{-1}\left(B_{n}\right)}$ and the selection lemma is proved.

REMARKS. (1) Note that in the situation of the lemma the inverse image $\{x \in X \mid$ $\Phi(x) \cap A \neq \varnothing\}$ of a closed set $A \subset Z$ under $\Phi$ need not be Baire measurable. Therefore, the theorem of Kuratowski and Ryll-Nardzewski applied to $\Phi$, in general only yields a Borel measurable selection for $\Phi$.

(2) The lemma, even in a slightly more general form, can also be derived from the main theorem in [2, Theorem 1, p. 343]. The proof given here uses methods similar to those employed in proving that general theorem.

4. Main results. In this section we will establish a selection theorem for correspondences whose graphs are groups. The main ingredients of the proof are the selection lemma and the fact that compact groups have the BSP.

THEOREM. Let $X$ and $Y$ be compact topological groups and $\Phi$ an u.s.c. compactvalued correspondence from $X$ to $Y$ such that $G(\Phi)$ is a subgroup of the product group $X \times Y$. Then $\Phi$ has a Baire measurable selection.

Proof. (a) First we consider the case $Y=\Pi_{i \in I} Y_{i}$, where each $Y_{i}$ is a compact metrizable group. For $J \subset I$ let $Y_{J}=\prod_{j \in J} Y_{j}$ and $\pi_{J}: Y \rightarrow Y_{J}, \hat{\pi}_{J}: X \times Y \rightarrow X \times Y_{J}$ be the canonical projections. Let $\Phi_{J}$ be the correspondence from $X$ to $Y_{J}$ defined by $\Phi_{J}(x)=\pi_{J}(\Phi(x))$. Then we have

$$
G\left(\Phi_{J}\right)=\hat{\pi}_{J}(G(\Phi))
$$

hence $G\left(\Phi_{J}\right)$ is a compact subgroup of $X \times Y_{J}$ because $\hat{\pi}_{J}$ is a continuous group homomorphism. In particular, $G\left(\Phi_{J}\right)$ has the BSP. Now let $\Gamma=\{(J, \varphi) \mid J \subset I$, $J \neq \varnothing, \varphi: X \rightarrow Y_{J}$ Baire measurable selection of $\left.\Phi_{J}\right\}$.

We introduce a partial order $\leqslant$ on $\Gamma$ by

$$
(J, \varphi) \leqslant(K, \psi) \text { iff } J \subset K \text { and } \pi_{j} \circ \varphi=\pi_{j} \circ \psi \text { for all } j \in J
$$

and claim that $\Gamma$ is nonempty and inductively ordered by $\leqslant$. For $i \in I$ the correspondence $\Phi_{i}$ is u.s.c. and takes compact values in the compact metrizable space $Y_{i}$. Hence, by the selection lemma, $\Phi_{i}$ admits a Baire measurable selectioin $\varphi_{i}$, i.e. $\left(\{i\}, \varphi_{i}\right) \in \Gamma$. Now let $\left(J_{\lambda}, \varphi_{\lambda}\right)_{\lambda \in \Lambda}$ be a chain in $\Gamma$. Let $J=\cup J_{\lambda}$ and define $\varphi: X \rightarrow Y_{J}$ by $\pi_{j} \varphi(x)=\pi_{j} \varphi_{\lambda}(x)$, if $j \in J_{\lambda}$.

Then $\varphi$ is a well-defined map. The definition of $\varphi$ and the Baire measurability of the $\varphi_{\lambda}$ 's implies that for each $j \in J$ the map $\pi_{j} \circ \varphi$ is Baire measurable. Since the Baire $\sigma$-algebra on $Y_{J}$ is the smallest $\sigma$-algebra rendering all the maps $\pi_{j}$ measurable, it follows that $\varphi$ is Baire measurable. Therefore $(J, \varphi)$ is an upper bound of $\left(J_{\lambda}, \varphi_{\lambda}\right)_{\lambda \in \Lambda}$ in $\Gamma$. By Zorn's lemma there exists a maximal element $(M, \mu)$ in $\Gamma$. To complete the proof of (a) it remains to show $M=I$. Assume the contrary. Then there is a $j \in I \backslash M$. Define a correspondence $\Psi$ from $G(\Phi)$ to $Y_{j}$ by

$$
\Psi((x, y))=\left\{z \in Y_{j} \mid(y, z) \in \Phi_{M \cup\{j\}}(x)\right\} \text {. }
$$

The graph of $\Psi$ is equal to $G\left(\Phi_{M \cup\{j\}}\right)$, hence compact. This implies that $\Psi$ is u.s.c. and compact-valued. Since $G\left(\Phi_{M}\right)$ has the BSP, the selection lemma yields a Baire measurable selection $\psi$ for $\Psi$. Define $\varphi: X \rightarrow Y_{M \cup(j)}$ by $\varphi(x)=(\mu(x), \psi(x, \mu(x)))$. 
Then $\varphi$ is obviously a selection for $\Phi_{M \cup\{j\}}$. To show that $\varphi$ is Baire measurable we have to check the measurability of the maps $\pi_{i}^{\circ} \varphi$ with $i \in M \cup\{j\}$. For $i \in M$ it follows from $\pi_{i} \circ \varphi=\pi_{i} \circ \mu$. Moreover, we have $\pi_{j} \varphi(x)=\psi(x, \mu(x))$ for all $x \in X$. Since $x \mapsto(x, \mu(x))$ is Baire measurable as a map into $X \times Y_{M}$ taking values in $G\left(\Phi_{M}\right)$, it is also Baire measurable as a map into $G\left(\Phi_{M}\right)$ because $G\left(\Phi_{M}\right)$ is compact. Hence $\pi_{j} \circ \varphi$ is Baire measurable as a composition of Baire measurable maps. Thus $(M \cup\{j\}, \varphi)$ is an element of $\Gamma$ strictly larger than the maximal element $(M, \mu)$, a contradiction.

(b) To prove the general case we observe that every compact topological group $Y$ is a subgroup of a product $\Pi Y_{i}$ of compact metrizable groups $Y_{i}$, because it is a projective limit of such groups (cf. e.g. Higgins [3, p. 98, Theorem $\left.A^{\prime \prime \prime}\right]$ ). Hence by (a) there exists a selection $\varphi$ of $\Phi$ which is Baire measurable as a map into $\Pi Y_{i}$. As before we see that it is also Baire measurable as a map into $Y$. Hence the theorem follows.

Important examples of correspondences satisfying the assumptions of our theorem are given by $\Phi=p^{-1}$ where $p$ is a continuous homomorphism from one compact group onto another. This immediately leads to the following corollary.

Corollary. Let $X$ and $Y$ be compact topological groups and $p: Y \rightarrow X$ a continuous surjective homomorphism. Then there exists a Baire measurable map $\varphi: X \rightarrow Y$ with $p \circ \varphi=\mathrm{id}_{X}$.

In particular the result announced in the introduction holds.

REMARK. The map $\varphi$ in the corollary can be chosen in such a way that it maps the identity element onto the identity element (define a new section by $x \mapsto \varphi(e)^{-1} \varphi(x)$ ). Therefore one always has measurable cross sections in the sense of Rieffel [7, p. 872], provided the groups involved are compact.

ACKNOWLEDGEMENT. The authors would like to thank J. Kupka for having brought this problem to their attention.

ADDED IN PROOF. Using the same methods, it can be shown that the answer to Kupka's question-mentioned in the introduction-remains "yes" even if the normality condition on the subgroup $H$ is dropped.

\section{REFERENCES}

1. M. Bockstein, Un théorème de séparabilité pour les produits topologiques, Fund. Math. 35 (1948), 242-246.

2. S. Graf, A measurable selection theorem for compact-valued maps, Manuscripta Math. 27 (1979), $341-352$.

3. P. J. Higgins, An introduction to topological groups, Cambridge Univ. Press, Cambridge, 1974.

4. J. Kupka, Strong liftings with application to measurable cross sections in locally compact groups (preprint), 1980.

5. K. Kuratowski and C. Ryll-Nardzewski, A general theorem on selectors, Bull. Acad. Polon. Sci. Sér. Sci. Math. 13 (1965), 397-403.

6. A. Pelczyński, Linear extensions, linear averagings, and their applications to linear topological classification of spaces of continuous functions, Dissertationes Math. (Rozprawy Mat.) 58 (1968).

7. M. A. Rieffel, On extensions of locally compact groups, Amer. J. Math. 88 (1966), 871-880.

Mathematisches Institut, Universitat Erlangen-NUrnberg, D-8520 Erlangen, Federal RePUBLIC OF GERMANY 